\title{
Mesoscale simulations of polymer dynamics in microchannel flows
}

\author{
L. Cannavacciuolo, R. G. Winkler and G. Gomprer
}

Institut für Festkörperforschung, Forschungszentrum Jülich, 52425 Jülich

PACS 47.61.-k - Micro- and nano-scale flow phenomena

PACS 47.57.Ng - Polymers and polymer solutions

PACS 83.50.- $-\mathrm{v}$ - Deformation and flow

PACS 82.20.Wt - Computational modelling; simulations

\begin{abstract}
The non-equilibrium structural and dynamical properties of flexible polymers confined in a square microchannel and exposed to a Poiseuille flow are investigated by mesoscale simulations. The chain length and the flow strength are systematically varied. Two transport regimes are identified, corresponding to weak and strong confinement. For strong confinement, the transport properties are independent of polymer length. The analysis of the long-time tumbling dynamics of short polymers yields non-periodic motion with a sublinear dependence on the flow strength. We find distinct differences for conformational as well as dynamical properties from results obtained for simple shear flow.
\end{abstract}

Introduction. - Confinement fundamentally alters the properties of dilute polymer solutions - compared to bulk behavior - when either the polymer radius of gyration is on the order of the characteristic dimensions of its proximity [1-4], and/or an external field is applied, e.g., - a shear or pressure field. In the first case, geometrical constraints lead to a stretching of the polymer parallel to the surfaces [1-6]. In the second case, in addition to flowinduced deformations, polymer-surface hydrodynamic interactions determine the polymer dynamics and leads to, e.g., cross-stream migration [7-12]. The migration effect has been studied intensively for DNA-like molecules by computer simulations and the relevance of hydrodynamic interactions has been confirmed. Experimental studies of individual DNA molecules in steady shear flow by fluorescence microscopy have provided a wealth of information on single polymer dynamics [13-16]. In particular, large conformational changes have been revealed due to tumbling motion $[15,17,18]$. Similar studies for flexible polymers confined between surfaces or in narrow channels have not been conducted so far, however, a similar complex dynamics can be expected. Understanding of single polymer behavior is of paramount importance for the emerging technology of microfluidic devices. Insight into the detailed microscopic conformational, dynamical, and transport properties of polymers, e.g., DNA, will help in the conception and design of such devices. Moreover, such studies will contribute to the understanding of the transport properties of biological macromolecules through blood vessels.
The proper account of hydrodynamic interactions is essential in simulation studies of fluid flows in channels as is emphasized by the appearance of cross-streamline migration. Recently developed mesoscale simulation techniques, such as Lattice Boltzmann simulations [12, 19], Brownian dynamics simulations with a hydrodynamic tensor [4], and multi-particle-collision dynamics [20,21] (also called stochastic rotation dynamics), are well suited for simulations of microchannels flows and are able to bridge the length- and time-scale gap among the solvent and solute degrees of freedom thereby taking hydrodynamic interactions adequately into account. In this letter, we will present results for the conformational and dynamical properties of polymers confined in a square channel and exposed to a Poiseuille flow by mesoscale computer simulations. Both, the polymer length as well as the pressure gradient are systematically varied. The considered polymers fall into the crossover regime from weak to strong confinement. Experiments [11] and simulations [8] predict a distinct different flow behavior in the two limits, which is confirmed by our simulations. The strong confinement regime has received less attention so far, it is however most relevant if microchannels are intended to be used for DNA characterization. In addition, we discuss chain orientation and tumbling dynamics, aspects which have not been addressed in previous studies.

Simulation method, model. - We use a hybrid simulation approach to study the properties of flexible polymers in flow, where molecular dynamics simulations 


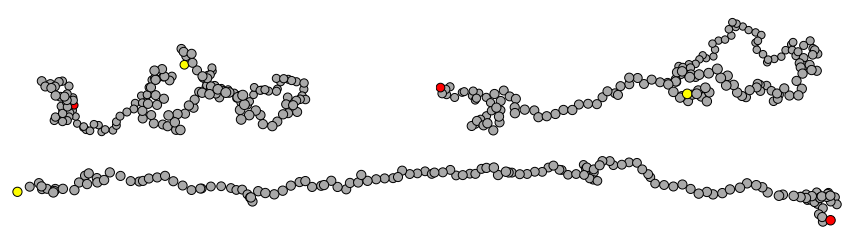

Fig. 1: Conformations of a polymer of length $N=160$ in a channel of width $L=15 a$ for weak (Peclet number $P e^{L}=10$, top two images) and strong flow $\left(P e^{L}=100\right.$, bottom). The latter yields close to fully stretched polymers.

(MD) are combined with the multi-particle-collision dynamics (MPC) method for the solvent $[22,23] . \quad$ MPC is particle based and proceeds in two steps. In the streaming step, the solvent particles of mass $m$ move ballistically for a time $h$. In the collision step, particles are sorted into the cells of a cubic lattice of lattice constant $a$ and their relative velocities, with respect to the center-of-mass velocity of each cell, are rotated around a random axis by an angle $\alpha$. For every cell, mass, momentum, and energy are conserved. The algorithm is described in detail in refs. [22, 24-27]. The fluid is confined in a square channel of side length $L$ and periodic boundary conditions are applied along the channel axis $\left(L_{\|}\right)$. No-slip boundary conditions are imposed by the bounce-back rule, as described in Ref. [28], and flow is induced by a gravitational force $(F=m g)$ acting on every fluid particle. The linear polymer is comprised of mass points of mass $M$, which are connected by a strong harmonic potential with an equilibrium bond length $b$. Excluded-volume interactions are taken into account by the well-known 12-6 truncated Lennard-Jones potential with the parameters $\sigma$ and $\epsilon$ [29]. Since we consider pressure-driven flows, no gravitational force acts on the polymer. The interaction of a polymer with the solvent is realized by inclusion of its monomers in the MPC collision step [30]. Extensive studies of polymer dynamics confirm the validity of this procedure $[20,26,29,30]$. Specifically, we employ the parameters $\alpha=130^{\circ}, h=0.1 \tau$, with $\tau=\sqrt{m a^{2} / k_{B} T}\left(k_{B}\right.$ is Boltzmann's constant and $T$ the temperature), the number of particles per cell $\rho=10, M=m \rho, b=\sigma=a$, the fluid mass density $\varrho=\rho m / a^{3}, k_{B} T / \epsilon=1$, and the time step in MD simulations $h_{M D}=5 \times 10^{-3} \tau$. Polymers with the monomer numbers $N=20,40,80$, and 160 are considered. To minimize self-interactions of periodic images, the corresponding channel lengths are $L_{\|}=40 a$, $60 a, 100 a$, and $170 a$, respectively. Simulations of the pure solvent confirm that the generated velocity profiles agree with the solution of the Navier-Stokes equations for the considered geometry.

Results. - We expect a pronounced different behavior of polymers in Poiseuille flow for radii of gyration either smaller or larger than the width of the channel [11]. Scaling considerations based on a blob model [1] and simulations [4] yield an equilibrium stretch of a flexible poly-

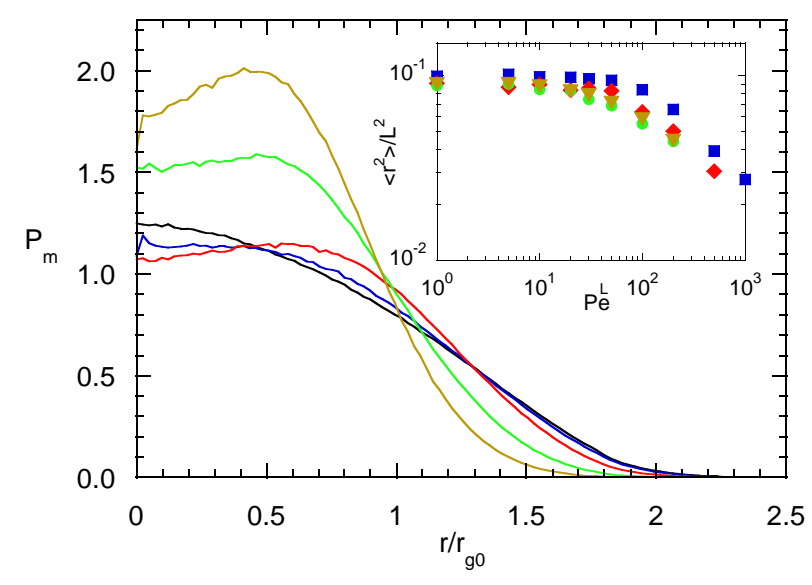

Fig. 2: Radial monomer distribution functions for the Peclet numbers $P e^{L}=0,10,50,100,200$ (top to bottom at $r / r_{g 0}=$ $1.5), N=40$, and $L / a=15$. Inset: widths of the distribution functions for the chain lengths $N=20$ (squares), $N=40$ (diamonds), $N=80$ (bullets), and $N=160$ (triangles).

mer in a microchannel when its bulk radius of gyration $r_{g 0} \sim b N^{3 / 5}$ exceeds the lateral channel dimension, i.e., for $r_{g 0} \gg L$. Here, the channel width becomes the characteristic length scale and the blob relaxation time, which is determined by $L$, will be the relevant time scale $[4,8]$. For $r_{g 0} \ll L$, the conformations are unaffected by confinement and the characteristic length and time scales are given by the bulk values of the polymer. The ratios $2 r_{g 0} / L$ for the considered polymer lengths and the channel width $L=15 a$ cover the range $0.36-1.3$, corresponding to the crossover regime between weak and strong confinement. The snapshots of fig. 1 illustrate the preferred average alignment of a polymer along the flow direction.

Figure 2 provides an example of the radial monomer distribution for the polymer length $N=40$ and various Peclet numbers. Here, we defined the Peclet number $P e^{L}$ as $P e^{L}=\dot{\gamma} \tau_{L}$, with an effective shear rate $\dot{\gamma}=g L \varrho /(2 \eta)$ and the relaxation time $\tau_{L}=\eta(L / 2)^{3} /\left(3 k_{B} T\right)$ ( $\eta$ is the solvent viscosity) [31], where the blob diameter has been replaced by the channel width. A similar approach has been adopted in ref. [8]. The distribution functions are normalized such that $\int_{0}^{\infty} P_{m}\left(r / r_{g 0}\right) r / r_{g 0}^{2} d r=1$. With increasing Peclet number, the distribution $P_{m}$ increases at $r / r_{g 0} \approx 1$ due to the stretching of the polymer by the flow. For even large $P e^{L}$, migration sets in and the maximum of $P_{m}$ shifts to smaller radii, as already observed in number of other studies $[7-10,12]$. The widths of the radial distribution functions

$$
\left\langle r^{2}\right\rangle=\int_{0}^{\infty} r^{3} P_{m}(r) d r
$$

for the various chain lengths are shown as inset in fig. 2 , For small Peclet numbers, the widths are independent of $P e^{L}$ and decay at large $P e^{L}$. There seems to be a weak dependence on chain length only. The widths start to decrease at approximately the same Peclet number. This 


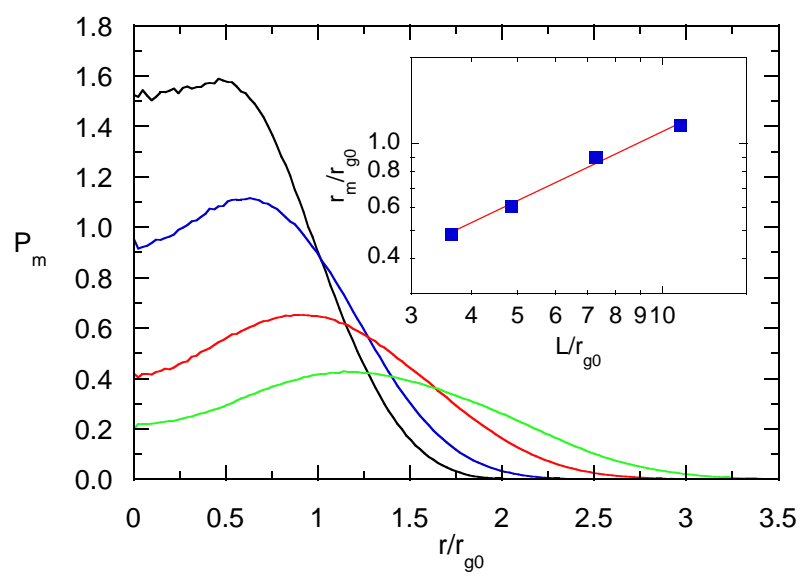

Fig. 3: Radial monomer distribution functions for the channel widths $L / a=15,20,30$, and 45 (left to right). The polymer length is $N=40$ and $g \tau^{2} / a=0.01$, i.e., the curvature of the flow profile is independent of $L$. Inset: position of the maximum as function of the channel width. The straight line represents the power law $\left(L / r_{g 0}\right)^{4 / 5}$.

is consistent with our assumption that the blobs determine the polymer properties. The approach to a universal limiting curve with increasing polymer length is explained as follows. In general, $P_{m}$ exhibits the dependence $P_{m}(b, r, L, N, g)$, and correspondingly the width $W^{2}=\left\langle r^{2}\right\rangle=W^{2}(b, L, N, g)$. Scaling all lengths by $L$ and using the above definition of the Peclet number yields the dependence $W^{2} / L^{2}=f\left(r_{g 0} / L, b N / L, P e^{L}\right)$. For strongly confined polymers, the width is determined by blobs - and thus by $L$ - rather than by the polymer length, hence, we find $W^{2} / L^{2}=f\left(P e^{L}\right)$, i.e., it depends on the Peclet number only. According to current understanding, migration is caused by polymer-surface hydrodynamic interactions [7-10,12]. Since hydrodynamic interactions are long-range in nature, it is difficult to unravel the system size dependence of migration. To study the influence of the channel width on migration, we performed simulations for various channel widths $L$ at the same curvature of the flow profile $\left(g \tau^{2} / a=0.01\right)$ and polymer length $N=40$. As shown in fig. 3. cross-streamline migration is well pronounced for all $L$, with a shift of the maximum of the monomer distribution to larger radii with increasing $L$. The position of the maximum $\left(r_{m}\right)$ exhibits the chainlength dependence $r_{m} \sim L^{4 / 5}$ as shown in the inset of fig. 3, i.e., migration is more pronounced for less strongly confined polymers. The changes in polymer conformations are reflected in their radii of gyration, which are displayed in fig. (4. Evidently, short polymers $(N<40)$ experience only minor conformational restrictions at equilibrium, whereas longer polymers are stretched along the channel and squeezed in the transverse direction. Beyond a certain Peclet number $P e_{c}^{L}$, flow induces or enhances stretching parallel to the channel and transverse shrinkage. This $P e_{c}^{L}$ value depends only very weakly on chain length.

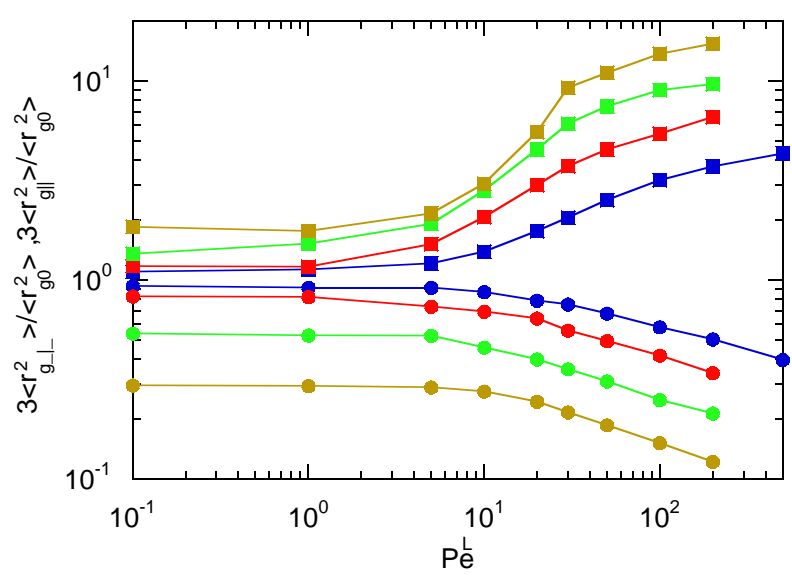

Fig. 4: Mean square radii of gyration parallel (squares) and transverse (bullets) to the channel for the chain lengths $N=$ 20, 40, 80, 160 (bottom to top, squares; top to bottom, bullets) and $L=15 a$ as a function of the Peclet number $P e^{L}$.

Figure 5 shows the component of the mean square radius of gyration along the channel axis for various chain lengths. Here, a different kind of Peclet number is employed, which is defined as $P e=\dot{\gamma} \tau_{G}$, with $\tau_{G}=\eta r_{g 0}^{3} /\left(3 k_{B} T\right)$ [31], i.e., the polymer relaxation time is used instead of a blob-related time. The Peclet number is then chain-length dependent. The shorter polymers seem to exhibit universal behavior for Peclet numbers up to $P e \approx 5$. For larger $P e$, finite polymer-size effects play a role. However, the longer polymers show a different behavior. This confirms our picture of the various involved length and time scales. The polymers of lengths $N<40$ are only weakly confined in the channel. The flow behavior is determined by the equilibrium radius of gyration and the longest polymer relaxation time. Instead of the effective shear rate $\dot{\gamma}=g L \varrho /(2 \eta)$, we could use a value defined in a different way, e.g., the local shear rate averaged over the channel width. For longer polymers $(N>40)$, the channel width defines the relevant length and time scales as long as the confinement is felt by the polymer. At high flow velocities, the polymer migrates to the channel center and is no longer in contact with the surfaces (cf. fig. 2).

So far, we have discussed conformational properties which are averaged over the microchannel. Due to the parabolic velocity profile, however, the local shear rate changes linearly with the radial distance. Thus, we can define a local Peclet number by $P e^{c m}=\varrho g r_{g 0}^{3} r_{c m} /\left(3 k_{B} T\right)$, where $r_{c m}$ is the radial chain center-of-mass position. Figure 6 shows the dependence of the mean square radius of gyration parallel to the channel as function of $P e^{c m}$ for the chain length $N=40$ and $L=15 a$. The various data sets cover the range of Peclet numbers $P e^{L}=1-200$. For small $P e^{c m}$, i.e., in the central part of the channel we find a stretching of the polymer by the flow field which increases with increasing field strength $P e^{L}$. Thus, in the central part, the channel flow is different from a simple shear flow - the extended polymer never experience a linear veloc- 


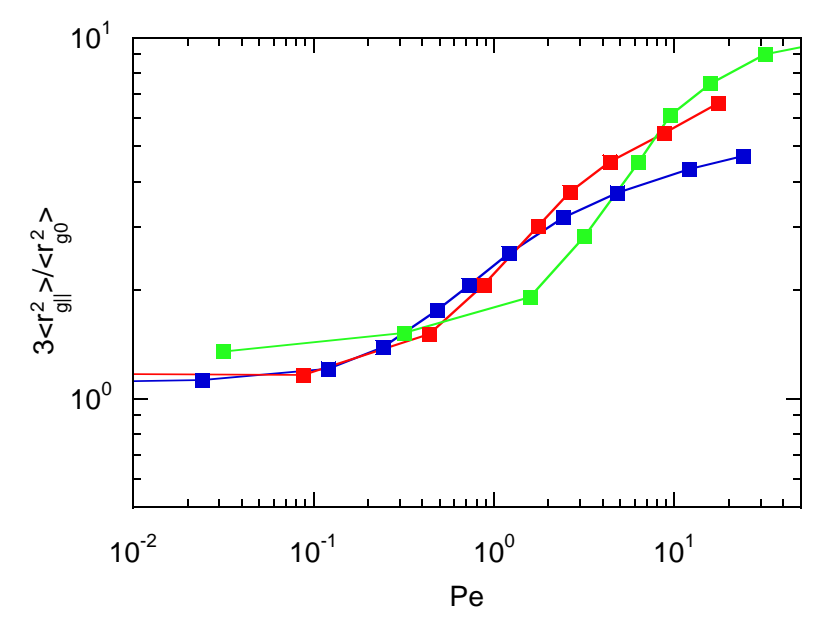

Fig. 5: Mean square radii of gyration parallel to the channel for the chain lengths $N=20,40$, and 80 (bottom to top) and $L=15 a$ as a function of the Peclet number $P e$.

ity profile only. At larger radial distances, the polymer is further stretched, particulary for larger $P e^{L}[9]$. More importantly, the various data sets seem to approach a limiting curve for sufficiently large $r_{\mathrm{cm}}$. However, this curve deviates from the theoretical dependence valid for linear shear flows, at least for the length range covered by our simulations. The transverse radius of gyration exhibits a similar deviation from the theoretical prediction. This leads us to the conclusion that the non-equilibrium structural properties of flexible polymers in a Poiseuille flow are even locally different from those of a polymer in linear shear flow at least as long as the characteristic chain dimensions are on the order of the channel width. The reason seems to be the variation of the relevant relaxation times as $P e^{L}$ and the center-of-mass position change.

In addition, we analyze the orientational distribution function of the end-to-end vector. Since the system is almost rotational symmetric with respect to the channel axis, only the distribution function $P_{a}(\theta)$, where $\theta$ $(0<\theta<\pi)$ is the angle between the end-to-end vector and the flow direction, needs to be studied. As shown in fig. 7 . the distribution is almost independent of the angle $\theta$ at small Peclet numbers. The deviation from the bulk value $1 / 2$ is caused by the confinement of the polymer. (The distribution is normalized such that $\int_{0}^{\pi} P_{a}(\theta) \sin \theta d \theta=1$.) As expected, there is no preferred angle; the average is $\langle\theta\rangle=0$. The shape of the distribution strongly depends on the Peclet number. For large Peclet numbers, $P_{a}$ decays algebraically as $\theta^{-2}$. A similar algebraic decay is found for the angle between the end-to-end vector and its projection onto the shear plane of a flexible polymer in shear flow $[16,18]$. However, the distribution $P_{a}$ also reflects the fundamental difference of polymers in Poiseuille and shear flow. For shear flow, there exists a regime of shear rates, where the distribution is Gaussian, only for large shear rates follows the algebraic decay $[16,18]$. In fig. 7 no Gaussian regime is present. Because of the nonlinear flow

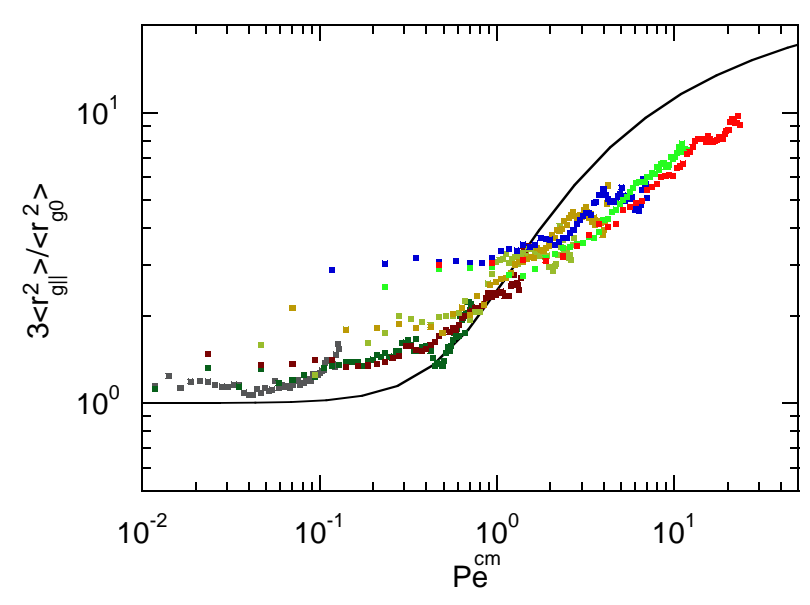

Fig. 6: Mean square radii of gyration parallel to the channel for the Peclet numbers $P e^{L}=1,5,10,20,30,50,100,200$ (left to right) as a function of the Peclet number $P e^{c m}$, where $P e^{c m} \sim r_{c m}$. The solid line is an analytical result for a chain of the same persistence length in simple shear flow [18].

profile, an algebraic decay is not necessarily surprising, compared to simple shear, where this fact is explained by higher order correlations and the importance of the whole time history. Nevertheless, the similarity is striking and might be traced back to the same origin. The radial width of the monomer and center-of-mass distributions decrease with increasing flow strength (cf. fig. 3) and a polymer spends more time in the region of higher axial fluid velocity [8]. This results in an increased polymer transport velocity compared to the average solvent velocity, as shown in fig. 8. For a wide channel $r_{g 0} \ll L$, the ratio of $v_{c m} / v_{s}$ is close to unity at equilibrium. Conformational restrictions for $r_{g 0} \ll L$ lead to a narrowing of the radial distribution functions [11] accompanied by increased polymer flow velocities even at very weak imposed flows. This expected transport behavior is shown in fig. 8. At small Peclet numbers $P e^{L} \lesssim 50$, we find - except for $N=20$ - center-of-mass velocities which depend only weakly on $P e^{L}$, caused by confinement. Only for the polymer of length $N=20$ reduces the velocity ratio to unity at equilibrium. With increasing Peclet number, the average polymer velocity increases, starting at about the same Peclet number $P e^{L}$ for all chain lengths, and the ratio reaches almost its maximum value of two. We obtain a chainlength dependence of the velocity ratio for short polymers only, which is rather a consequence of the weaker confinement of the shorter polymer than a true molecular weight dependence. The ratios for the longer polymers seem to approach a universal limiting curve, which is consistent with the assumption that local properties on a blob scale rather than global polymer properties determine their behavior. As a consequence, no separation of highly confined DNA according to molecular weight is possible. Polymers in simple shear flow exhibit large conformational changes due to tumbling motion, i.e., they stretch and recoil in the 


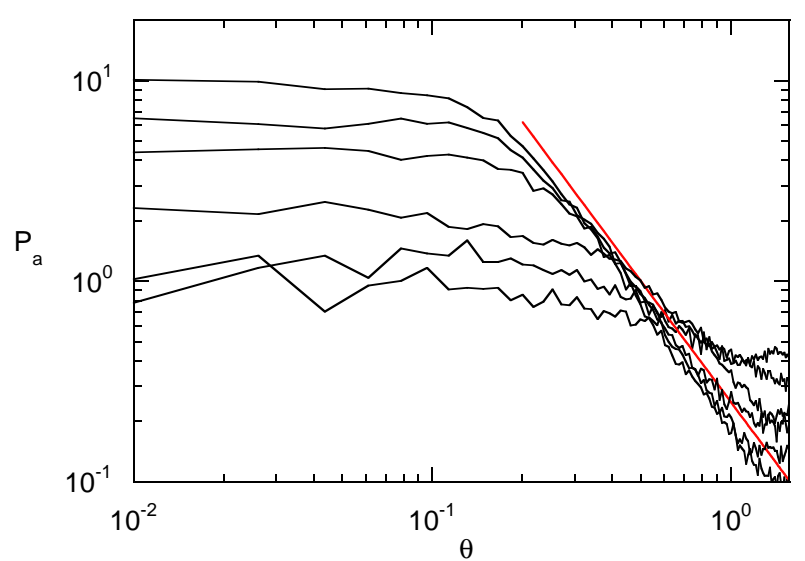

Fig. 7: Probability distribution of the angle between the endto-end vector and the flow direction for the Peclet numbers $P e^{L}=1,5,10,30,50,100$ (bottom to top) and the channel width $L=15 a$. Only half of a period is shown. The solid line represents the power-law dependence $P_{a} \sim \theta^{-2}$.

course of time [15-18]. Visual inspection of the polymer dynamics in Poiseuille flow reveals similar large conformational fluctuations (cf. fig. 1). Tumbling is characterized by a typical time denoted as tumbling time $t_{T}$. There are various ways to extract such a characteristic time, although the dynamics is non-periodic. We tried various strategies and found the following approach most useful. We first determined the distribution of times between consecutive crossings of the end-to-end vector with the plane perpendicular to the flow direction, i.e., $\theta= \pm \pi / 2$. As shown in the inset of fig. 9, this yields exponentially decaying distributions $\left(P_{T} \sim e^{-t / t_{T}}\right)$ at large times. The extracted tumbling times $t_{T}$ are presented in fig. 9. A power-law fit yields the dependence $t_{T} \sim g^{-0.52}$. Simulations and analytical calculations [15-18] yield the dependence $t_{T} \sim \dot{\gamma}^{-2 / 3}$ for flexible polymers in linear shear flow. Hence, the tumbling times of polymers in Poiseuille flow exhibit a weaker dependence on the strength of the external field than in shear flow.

Conclusions. - We have analyzed the flow behavior of flexible polymers confined in a square channel with a side length comparable to the polymer radius of gyration. We have identified two distinct transport regimes were polymers behave similarly. For $r_{g 0} \ll L$ the polymers are unaffected by the channel geometry and the characteristic length and time scales are given by the radius of gyration and the longest polymer relaxation time, respectively. In the strongly confined regime $r_{g 0} \gg L$, the polymer structure and dynamics are determined by blobs with a size of the channel cross section. Polymers with $r_{g 0} \approx L / 2$ show a complicated and non-universal behavior. We expect that the properties of polymers in a slit geometry are less severely affected by confinement than those of polymers in a channel. In the slit geometry, the polymer is always able to relax in the direction parallel to the surfaces and hence the longest relaxation time is always re-

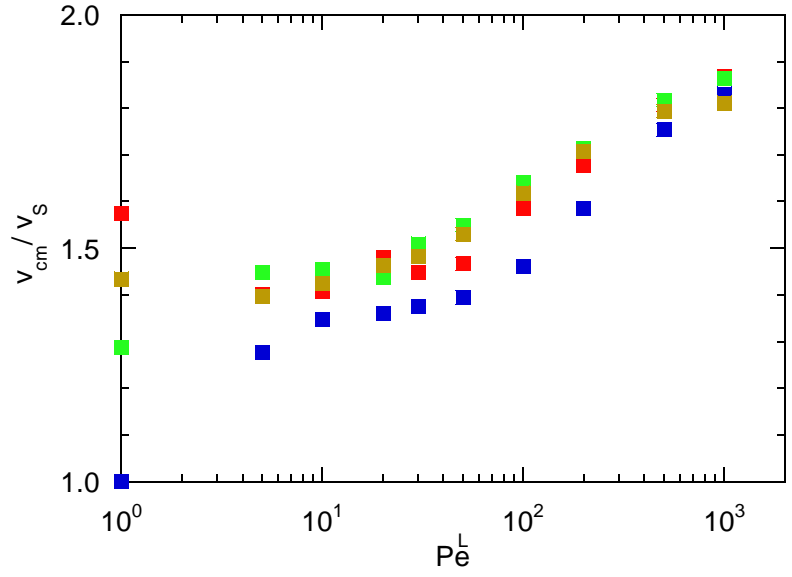

Fig. 8: Ratio of the polymer center-of-mass velocity $v_{c m}$ and the average solvent velocity $v_{s}$ for the chain lengths $N=20$ (blue), 40 (red), 80 (green), and 160 (orange) and the channel width $L=15 a$.

lated to the polymer length. The analysis of the radial polymer deformation, the distribution of the orientation angle, and the tumbling times reveals differences among Poiseuille flow and simple shear flow for the strongly confined polymers.

\section{REFERENCES}

[1] Wall F. T., Seitz W. A., Chin J. C. and de Gennes P. G., Proc. Natl. Acad. Sci. USA, 75 (1978) 2069.

[2] Brochard F. and de Gennes P. G., J. Chem. Phys., 67 (1977) 52.

[3] van Vliet J. H. and ten Brinke G., J. Chem. Phys., 93 (1990) 1436.

[4] Jendrejack R. M., Dimalanta E. T., Schwartz D. C., Graham M. D. and de Pablo J. J., Phys. Rev. Lett. , 91 (2003) 038102.

[5] Jendrejack R. M., Schwartz D. C., Graham M. D. and de Pablo J. J., J. Chem. Phys., 119 (2003) 1165.

[6] Tegenfeldt J. O., Prinz C., Cao H., Reisner W. W., Riehn R., Wang Y. M., Cox E. C., Sturm J. C., SilBerzan P. and Austin R. H., Proc. Natl. Acad. Sci. USA , 101 (2004) 10979.

[7] Agarwal U. S., Dutta A. and Mashelkar R. A., Chem. Eng. Sci., 49 (1994) 1693.

[8] Jendrejack R. M., Schwartz D. C., de Pablo J. J. and Graham M. D., J. Chem. Phys., 120 (2004) 2513.

[9] Usta O. B., Butler J. E. and Ladd A. J. C., Phys. Fluids, 18 (2006) 031703.

[10] Khare R., Graham M. D. and de Pablo J. J., Phys. Rev. Lett., 96 (2006) 224505.

[11] Stein D., van der Heyden F. H. J., Koopmans W. J. A. and Dekker C., Proc. Natl. Acad. Sci. USA, 103 (2006) 15853.

[12] Usta O. B., Butler J. E. and Ladd A. J. C., Phys. Rev. Lett., 98 (2007) 098301.

[13] Sмiтн D. E., BABсоск H. P. and Chu S., Science, 282 (1999) 1724. 


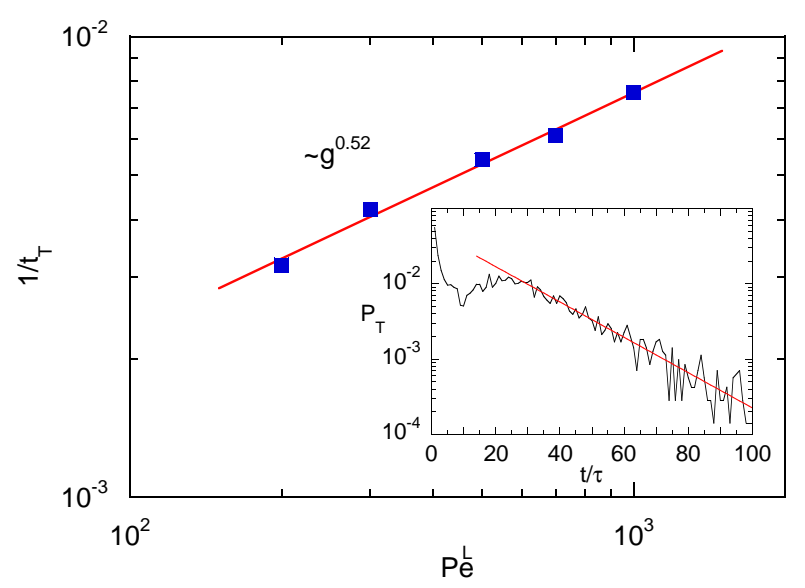

Fig. 9: Tumbling times for the chain length $N=20$ and the channel width $L=15 a$. Inset: Distribution of tumbling times for $P e^{L}=500$.

[14] LeDuc P., Haber C., Boa G. and Wirtz D., Nature, 399 (1999) 564.

[15] Schroeder C. M., Teixeira R. E., Shaqfen E. S. G. and Chu S., Phys. Rev. Lett., 95 (2005) 018301.

[16] Gerashchenko S. and Steinberg V., Phys. Rev. Lett. , 96 (2006) 038304.

[17] Delgado-Buscalioni R., Phys. Rev. Lett. , 96 (2006) 088303.

[18] Winkler R. G., Phys. Rev. Lett. , 97 (2006) 128301.

[19] Ahlrichs P. and Dünweg B., J. Chem. Phys., 111 (1999) 8225.

[20] Webster M. A. and Yeomans J. M., J. Chem. Phys., 122 (2005) 164903.

[21] Noguchi H. and Gompper G., Proc. Natl. Acad. Sci. USA, 102 (2005) 14159.

[22] Malevanets A. and Kapral R., J. Chem. Phys. , 110 (1999) 8605.

[23] Ihle T. and Kroll D. M., Phys. Rev. E , 63 (2001) 020201(R).

[24] Kikuchi N., Pooley C. M., Ryder J. F. and Yeomans J. M., J. Chem. Phys., 119 (2003) 6388.

[25] Tüzel E., Strauss M., Ihle T. and Kroll D. M., Phys. Rev. E , 68 (2003) 036701.

[26] Ripoll M., Mussawisade K., Winkler R. G. and Gompper G., Europhys. Lett. , 68 (2004) 106.

[27] Ripoll M., Mussawisade K., Winkler R. G. and Gompper G., Phys. Rev. E, 72 (2005) 016701.

[28] Lamura A., Gompper G., Ihle T. and Kroll D. M., Europhys. Lett. , 56 (2001) 319.

[29] Mussawisade K., Ripoll M., Winkler R. G. and Gompper G., J. Chem. Phys., 123 (2005) 144905.

[30] Malevanets A. and Yeomans J. M., Europhys. Lett. , 52 (2000) 231.

[31] Dor M. and Edwards S. F., The Theory of Polymer Dynamics (Clarendon Press, Oxford) 1986. 